\title{
Bacterial Vaccine
}

National Cancer Institute

\section{Source}

National Cancer Institute. Bacterial Vaccine. NCI Thesaurus. Code C96390.

A vaccine derived from bacteria whether killed, attenuated or live that is used for prophylaxis or treatment against infection. 